\title{
Deep optical imaging of the dark galaxy candidate AGESVC1 $282^{\star}$
}

\author{
Michal Bílek ${ }^{1}$, Oliver Müller ${ }^{1}$, Ana Vudragović ${ }^{2}$, and Rhys Taylor $^{3}$ \\ 1 Université de Strasbourg, CNRS, Observatoire Astronomique de Strasbourg (ObAS), UMR 7550, 67000 Strasbourg, France \\ e-mail: bilek@astro.unistra.fr \\ 2 Astronomical Observatory, Volgina 7, 11060 Belgrade, Serbia \\ 3 Astronomical Institute of the Czech Academy of Sciences, Boční II 1401/1a, 14100 Praha 4, Czech Republic
}

Received 13 August 2020 / Accepted 21 September 2020

\begin{abstract}
The blind HI survey Arecibo Galaxy Environment Survey (AGES) detected several unresolved sources in the Virgo cluster, which do not have optical counterparts in the Sloan Digital Sky Survey. The origin of these dark clouds is unknown. They might be crucial objects since they could be the so-called dark galaxies, that is, the dark matter halos without stellar content that are expected from cosmological simulations. In order to reveal the nature of the dark clouds, we took a deep optical image of one them, AGESVC1 282, with the newly-commissioned $1.4 \mathrm{~m}$ Milanković Telescope. After observing it for $10.4 \mathrm{~h}$ in the $L$-filter, the image reached a surfacebrightness limit of about $29.1 \mathrm{mag} \operatorname{arcsec}^{-2}$ in $V$. No optical counterpart was detected. We placed an upper limit on the $V$-band luminosity of the object of $1.1 \times 10^{7} L_{\odot}$, giving a stellar mass below $1.4 \times 10^{7} M_{\odot}$ and a HI-to-stellar mass ratio above 3.1 . By inspecting archival HI observations of the surrounding region, we found that none of the standard explanations for optically dark HI clouds fits the available constraints on this object.
\end{abstract}

Key words. galaxies: individual: AGESVC1 282 - galaxies: formation - techniques: image processing - galaxies: interactions galaxies: structure - galaxies: clusters: intracluster medium

\section{Introduction}

Recently, there has been a rise of interest in the low surface brightness (LSB) universe. While the existence of LSB galaxies has been long established (e.g., Sandage \& Binggeli 1984; Bothun et al. 1987); in the last few years, it has become possible to detect them in large numbers (e.g., van Dokkum et al. 2015; Koda et al. 2015; Venhola et al. 2017; Müller et al. 2017; Román \& Trujillo 2017; Greco et al. 2018; Prole et al. 2019; Habas et al. 2020). Given the difficulties in detecting these faint objects, it is natural to ask whether there might be a significant population of even fainter galaxies with perhaps no stellar content at all (Collins et al. 2020). The existence of dark matter halos without stellar content is the standard way to explain the missing satellites problem of cosmological dark-matteronly simulations (Simon \& Geha 2007; Sawala et al. 2016; Simpson et al. 2018).

One proposed way to detect such "dark galaxies" is through HI surveys. Indeed, there appears to be a gas density threshold for star formation (see Davies et al. 2006 for a detailed discussion). While numerous dark clouds, that is to say HI sources without optical counterparts, have been detected, establishing their nature is often difficult: HI removed through tidal encounters or ram pressure stripping might have a similar morphology and kinematics as dark galaxies (Duc \& Bournaud 2008). However, there is a set of objects in the Virgo cluster that does not appear to be explicable by tidal encounters (Taylor et al. 2017) or through interactions with the intracluster medium (Taylor et al. 2018). Their velocity widths are consistent with a significant

\footnotetext{
* Data underlying to Fig. 1 are only available at the CDS via anonymous ftp to cdsarc.u-strasbg. fr (130.79.128.5) or via http:// cdsarc.u-strasbg.fr/viz-bin/cat/J/A+A/642/L10
}

dark matter content, and simulations have shown that such dark galaxies would be able to withstand a disruption by other cluster members (Taylor et al. 2016).

These objects were first reported in Taylor et al. $(2012,2013)$ as part of the Arecibo Galaxy Environment Survey (AGES). Their radial velocities $\left(1000-2000 \mathrm{~km} \mathrm{~s}^{-1}\right)$ and distribution on the sky suggest that they are members of the Virgo cluster (in the case of AGESVC1 282 in the B cloud, at a distance of $23 \mathrm{Mpc}$, Gavazzi et al. 1999). Their HI masses are $\sim 1-3 \times 10^{7} M_{\odot}$, which is consistent with other tidal debris; however, their combination of isolation (>100 kpc from the nearest galaxy) and high line widths $\left(\sim 150 \mathrm{~km} \mathrm{~s}^{-1}\right)$ make them more unusual. Tidal debris is known to be found at large distances from its parent galaxy (e.g., Hess et al. 2017; Leisman et al. 2016; Serra et al. 2015), but with line widths typically $<50 \mathrm{~km} \mathrm{~s}^{-1}$. In contrast, high line width features are known to exist within large HI streams (e.g., Koopmann et al. 2008; Kent et al. 2009), but they are rarely found without a clear association to a likely parent galaxy. In this particular respect, the AGES Virgo clouds are highly unusual. In addition, their low mass and high velocity widths make them strongly deviant from the baryonic Tully-Fisher relation (BTFR, McGaugh 2005) if at the distance of the Virgo cluster. In order to reconcile them with the usual BTFR, they would need distances of $\sim 2 \mathrm{Mpc}$, but there are no other known foreground galaxies in the direction of the Virgo cluster. Analogous objects have not been detected in other galaxy clusters.

In this Letter, we describe extremely deep optical imaging to try to detect the optical counterpart of one of these dark clouds, AGESVC1 282 . This can potentially bring several possible benefits (see Sect. 3 and also Minchin et al. 2007): we might discover that the object has a faint galaxy as an optical counterpart (as in Impey et al. 1990 or Mihos et al. 2018), that the gas has been 
displaced from a faint parent galaxy that is much closer to the cloud than to the known brighter objects (as in the case of the HI cloud near VCC 1249, Arrigoni Battaia et al. 2012), or, alternatively, we might find stellar tidal debris that would indicate a tidal origin of the clouds. No optical counterpart was actually detected. The luminosity of the object must be below $1.1 \times 10^{7} L_{\odot}$. In this Letter, we assumed $H_{0}=71 \mathrm{~km} \mathrm{~s}^{-1} \mathrm{Mpc}^{-1}$ for consistency with earlier AGES papers, and we assume a distance to the object of $23 \mathrm{Mpc}$.

\section{Observations and data reduction}

The HI observations and data reduction of AGES are extensively described in Auld et al. (2006), Taylor et al. (2012, 2013). In brief, AGES is a drift scan survey with a sensitivity of $0.7 \mathrm{mJy}$, a spatial resolution of $3.5^{\prime}$, and a spectral resolution of $10 \mathrm{~km} \mathrm{~s}^{-1}$ after Hanning smoothing. This gives an approximate mass sensitivity of $8 \times 10^{6} M_{\odot}$ at the Virgo distance with a physical resolution of $23 \mathrm{kpc}$ in the $\mathrm{B}$ cloud of the cluster. Objects in AGES were detected through a combination of visual and automated source extraction procedures, and they were confirmed with follow-up observations using Arecibo's L-wide receiver. Eight optically dark clouds were discovered in Virgo by AGES. We selected AGESVC1 282 as our target (coordinates $\mathrm{RA}=12^{\mathrm{h}} 25^{\mathrm{m}} 24.10^{\mathrm{s}}$, Dec $=+08^{\circ} 16^{\prime} 54^{\prime \prime}$.0) because it has one of the highest HI signal-to-noise ratios (11.4) and velocity widths (W20 $=164 \mathrm{~km} \mathrm{~s}^{-1}$ ), though these features are similar to those of the other clouds. The width of the Arecibo beam puts an upper limit on the HI radius of AGESVC1 282 of 105". The lower limit on the HI radius is harder to establish, but it is likely higher than about $1 \mathrm{kpc}\left(10^{\prime \prime}\right)$; otherwise, the column density would exceed that of bright, star-forming galaxies (Taylor et al. 2016). Nevertheless this calculation is necessarily simplistic since predicting the optical brightness of the clouds is highly nontrivial (e.g., Bacchini et al. 2019). The HI mass of AGESVC1 282 was estimated as $10^{7.64} M_{\odot}$ (Taylor et al. 2012).

The target was observed during the two nights of 17 and 18 April 2020 with the 1.4 meter Milanković telescope equipped with an Andor IKONL CCD camera from the Astronomical Station Vidojevica (Serbia). Several images were taken with a $90^{\circ}$ camera rotation. Sky flat field images were taken for both regular and rotated camera positions. A total of 125 images were taken in the luminance $L$-filter with a $300 \mathrm{~s}$ exposure each. The integrated exposure time was $10.4 \mathrm{~h}$. The nights were clear with a $\sim 1 \mathrm{~h}$ period of thin cirrus. We measured an average image quality of 1 '.4. Utilizing the focal reducer delivers a square field-of-view with sides of $13: 3$ with a pixel scale of 0.39 pixels per arcsec. We applied a large dithering pattern randomized within a maximum offset of $4.5^{\prime}$, which is crucial when digging deep into the low-surface brightness regime at the 28-29 mag $\operatorname{arcsec}^{-2}$ level (Slater et al. 2009; Mihos 2019) and beyond (Trujillo \& Fliri 2016). Such a strategy provides a strong handle on the background subtraction, as well as on the removal of systematics arising from the instruments and observations, that is, from reflections or flat-fielding imperfections.

The calibration of the images follows the standard procedure as described in Müller et al. (2019) and includes bias, dark, flat, and illumination corrections with a careful customized background subtraction. The python pipeline implementing this calibration can be found online ${ }^{1}$. To create our final scientific image, we stacked all calibrated science frames with SWarp

\footnotetext{
1 gitlab.com/VoltarCH/milankovic-telescope-reductionpipeline
}

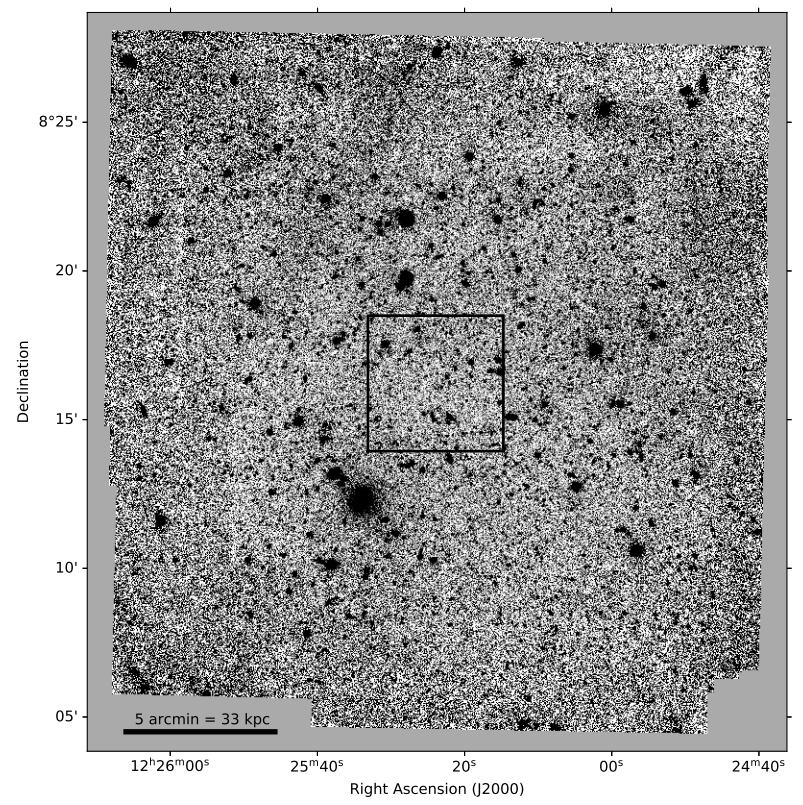

Fig. 1. Fully stacked and calibrated image. The image is centered on the dark cloud AGESVC1 282. The field-of-view is $21: 9 \times 22 ! 7$. The box indicates the stamps presented in Fig. 2 . The black line shows the scale at an assumed distance of $23 \mathrm{Mpc}$.

(Bertin 2010), where we used a median for stack. The final, fully calibrated, and dithering image reached a field-of-view of $21: 9 \times 22$ ! 7 , see Fig. 1 . The astrometry in the science frames was solved locally with the API of astrometry.net (Lang et al. 2010).

The zero point of this stacked image was estimated using the Sloan Digital Sky Survey (SDSS) DR12 star catalog (Alam et al. 2015). We used Source Extractor to measure isophotal magnitudes of the stars in the $L$-band. Since this catalog does not list stellar magnitudes in the $L$ filter, but instead the SDSS $g$ - and $r$-band magnitudes, we follow Javanmardi et al. (2016), who applied a linear transformation between the $L$ - and the $r$-band, thus taking into account a minor dependence on the $g-r$ color (see their Fig. 1). In cross-matching our instrumental magnitudes to the SDSS DR12 catalog, we found 350 stars in common that are brighter than 21 magnitude in the $g$-band. After the exclusion of stars that are too bright (saturated), we ended up with 336 stars. Furthermore, we decided to transform our magnitudes from the $g$ - and $r$-bands to the $V$-band using the transformations from Jester et al. (2005). This procedure yielded an intercept of $31.6 \pm 0.1 \mathrm{mag}$ in the $V$-band and a slope of $1.03 \pm 0.01$ in the $\mathrm{AB}$ system. From the standard deviation in 30 boxes with sizes of $10 \times 10 \operatorname{arcsec}^{2}$, we estimated a $1 \sigma$ surface brightness limit of $29.05 \pm 0.04 \mathrm{mag} \mathrm{arcsec}^{-2}$ in the $V$-band. The error of this limit was estimated by the standard deviation of the measured surface brightnesses of the different boxes.

To test the detectability of a LSB galaxy at the position of AGESVC1 282, we injected a range of artificial dwarf galaxies into the image (see Fig. 2). For this purpose, we modeled them with a 2D Sérsic profile with different effective radii and surface brightnesses. We used a Sérsic index of $n=0.6$, which is typical for faint dwarf galaxies (e.g., Müller et al. 2017; Venhola et al. 2017). Zero ellipticity was assumed. We used as maximum effective radius of $6 \mathrm{kpc}$, that is, the size of the most extended lowluminosity galaxies (Mihos et al. 2015). For the lower limit of the size of the stellar body, we used the lower limit on the size of the HI extent. In Fig. 2, we present a grid of such artificial galaxies. It is evident that in starting from an effective 


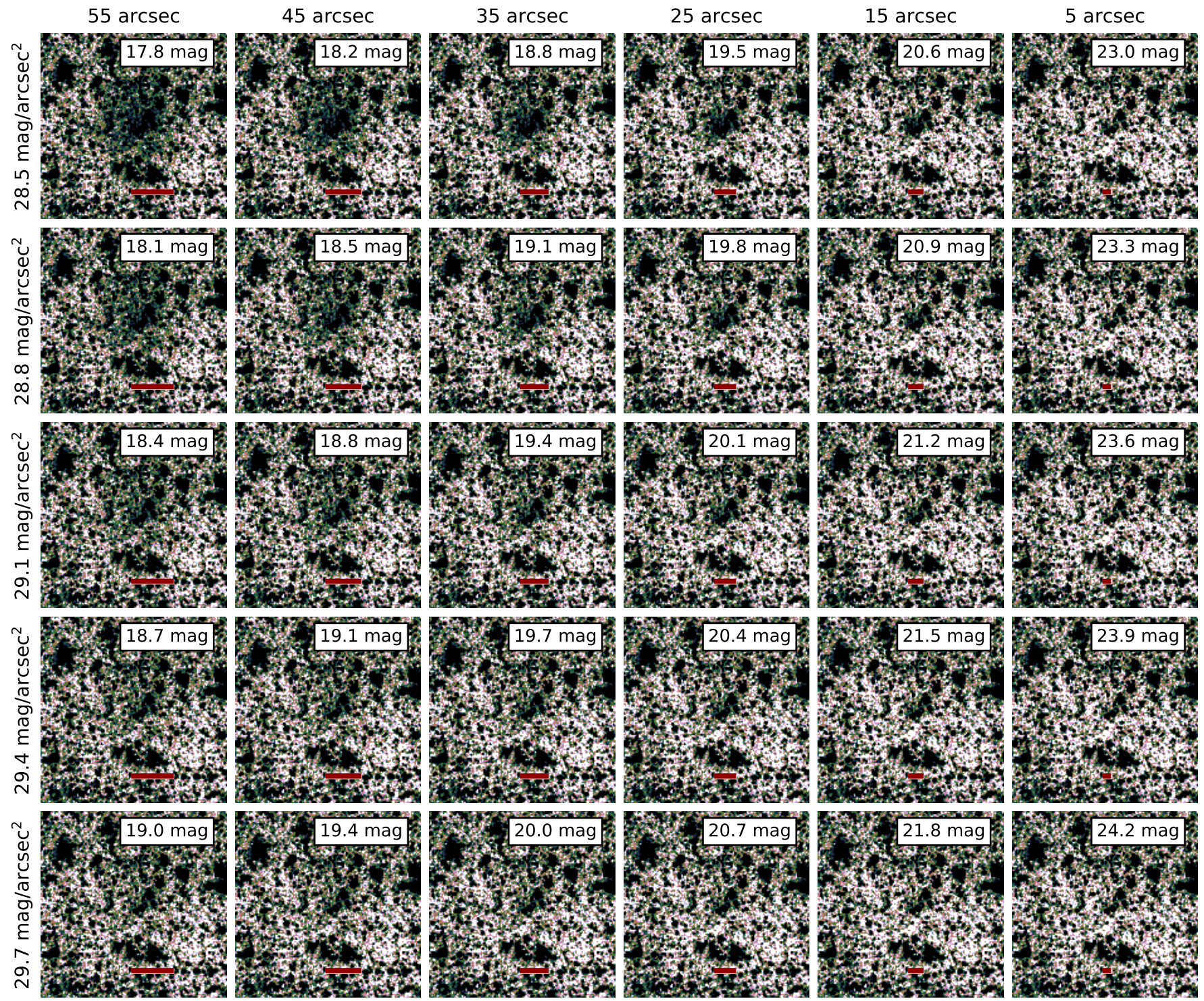

Fig. 2. Injected artificial dwarf galaxies. The effective radius and the effective surface brightness are varied in the horizontal direction and the vertical direction, respectively. The red line indicates the effective radius for each artificial galaxy. The number in the box represents the total apparent magnitude of the artificial galaxy. The images are smoothed with a Gaussian kernel with $\sigma=2 \mathrm{px}$ to enhance the LSB features. The brightness of the stamps is represented with a cube helix color scheme (Green 2011).

surface brightness of $29.1 \mathrm{mag} \operatorname{arcsec}^{-2}$ in the $V$-band, the artificial dwarf galaxies become indistinguishable from the background. This is similar to the surface brightness limit we estimated previously.

Using Fig. 2, we estimated that a reasonable upper limit on the apparent $V$-band magnitude of the dark cloud is $19.0 \mathrm{mag}$. We neglected the small uncertainty of the photometric calibration, as well as the foreground extinction of $0.06 \mathrm{mag}$ given by the NASA/IPAC Extragalactic Database ${ }^{2}$. At the adopted distance, the $V$-band luminosity of the dark cloud is estimated to be below $1.1 \times 10^{7} L_{\odot}$

In order to estimate the upper limit on the stellar mass of AGESVC1 282, we have to multiply the upper limit on the luminosity by the upper limit on the stellar mass-to-light ratio $(\mathrm{M} / \mathrm{L})$. We derived the latter from the work of Herrmann et al. (2016). These authors published M/Ls for 34 well-studied gas-rich dwarf irregular galaxies from the LITTLE THINGS

https://ned.ipac.caltech.edu/ sample. When estimating the mass of AGESVC1 282, we used the highest $V$-band $\mathrm{M} / \mathrm{L}$ that they encountered in their sample, that is to say 1.3. This led us to the upper limit on the stellar mass of AGESVC1 282 of $1.4 \times 10^{7} M_{\odot}$ and to the lower limit on the HI-to-stellar mass ratio of 3.1. As a consistency check, we used the catalog of pressure supported, gas-poor objects from Dabringhausen \& Fellhauer (2016) for objects with $L<1.1 \times 10^{7} L_{\odot}$. Out of these objects, $90 \%$ have a M/L below 3 , giving a lower estimate of the HI-to-stellar mass ratio for AGESVC1 282 of 1.3.

\section{Discussion and conclusions}

The dark cloud AGESVC1 282 is one of several HI sources associated with the Virgo cluster without optical counterparts in public surveys. The nature of these sources is still unclear. In order to further constrain the nature of these objects, we undertook very deep imaging of AGESVC1 282. By not detecting its optical counterpart, we found that the optical luminosity of the object 


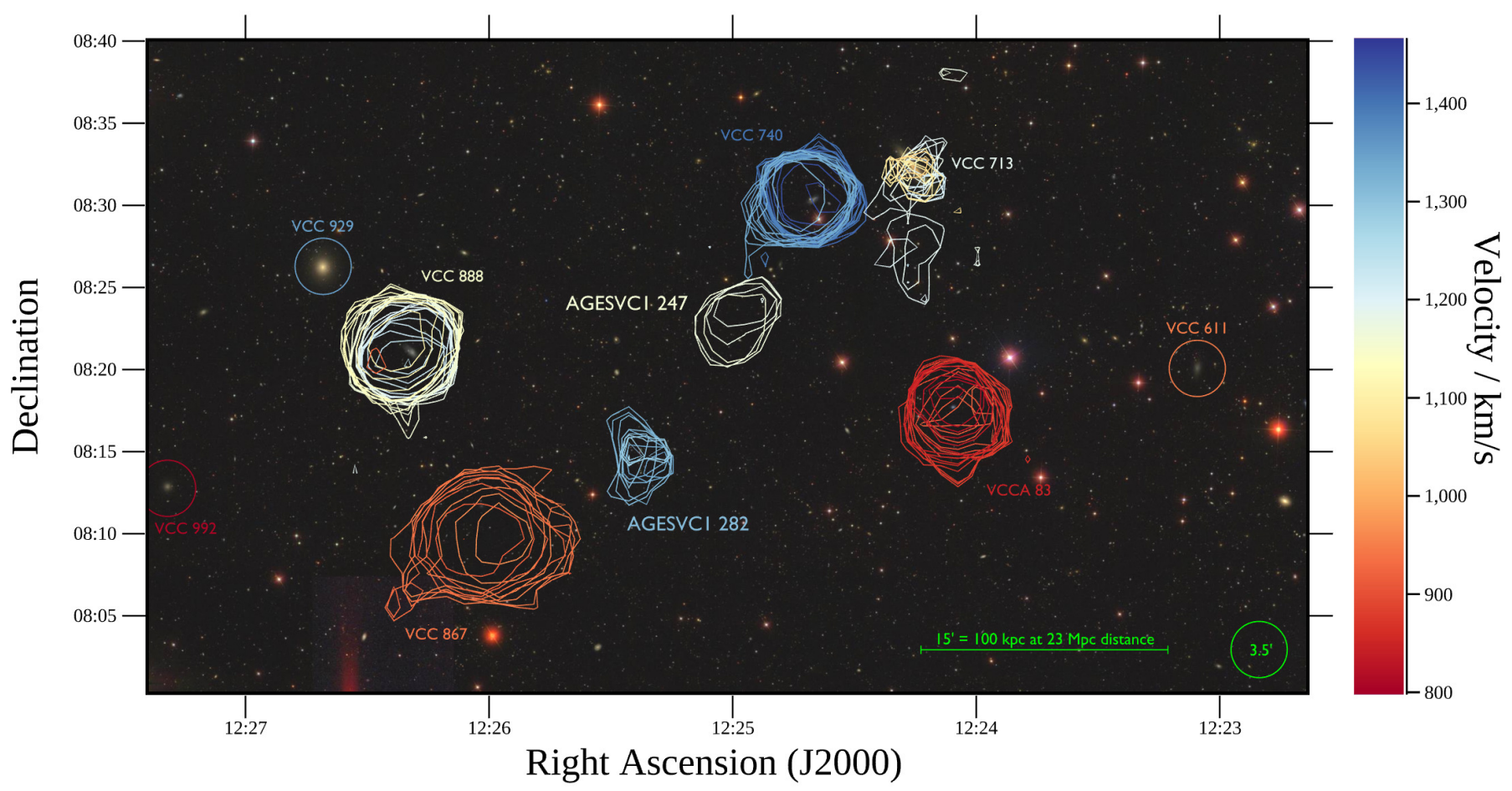

Fig. 3. Renzogram of the region surrounding AGESVC1 282, overlaid on an optical image from the SDSS. Each contour is at $4 \sigma$ (where the typical rms is $0.6-0.7 \mathrm{mJy}$ ), with the color indicating the velocity (channel width is $5.5 \mathrm{~km} \mathrm{~s}^{-1}$ ). Galaxies undetected in HI are marked with circles of the same size as the Arecibo beam.

is below $1.1 \times 10^{7} L_{\odot}$. This can be helpful as a constraint for the theoretical models of the formation of the dark clouds or for planning future observations. With the aim to help with the development of the theories of origin of the dark clouds, we briefly discuss several of them for the specific case of AGESVC1 282 on the basis of the HI observations by AGES.

Tidal debris. Figure 3 shows the HI contours in the region surrounding AGESVC1 282, which are overlaid on an SDSS optical image. Several candidate parent galaxies are visible, though (arguably) none are particularly convincing. The two most promising are VCC 740 and VCC 713 (see Taylor et al. 2020 for full details). VCC 740 shows a hint of an extension toward AGESVC1 282 and it is at a similar radial velocity (66 $\mathrm{km} \mathrm{s}^{-1}$ difference), though the extension is only barely visible in one channel. VCC 713 has a much clearer extension and the mass of $\mathrm{HI}$ in the extension is comparable to that in AGESVC1 282. The velocity difference of $195 \mathrm{~km} \mathrm{~s}^{-1}$ is compatible with tidal encounters, and while the projected separation of $153 \mathrm{kpc}$ would make this a large tidal system, it would not be exceptional.

While tidal debris is a promising explanation, several difficulties remain (see Taylor et al. 2016 for an extensive discussion). As discussed, the lack of a connecting stream between AGESVC1 282 and its parent galaxy is observationally unusual given its high line width. Moreover, the simulations of Taylor et al. (2016, 2017), looking for clouds matching these parameters, formed through multibody tidal encounters, while omitting the intercluster medium disfavors this scenario. That is to say clouds which are both isolated and of a high line width are rare according to the models, and those few that were formed in the usual manner had parent galaxies with much longer detectable HI streams than any seen in this region. We also note that AGESVC1 282 is one of six similar features that has been detected by AGES. Finally, the simulated clouds did not deviate as far from the from the BTFR as the observed features.

Dark or low-luminosity galaxy. The cold dark matter models of galaxy formation predicts the existence of "dark galaxies" with a negligible stellar content. Taylor et al. (2016) simulated the evolution of a dark galaxy corresponding to the observed clouds within the Virgo cluster. They showed that such an object would be strongly resistant to the effect of galaxy harassment on timescales of a few gigayears and, unlike clouds produced by tidal encounters, could maintain their deviation from the BTFR for similar periods. On the other hand, the simulations did not include the inter-cluster medium (ICM) whose effects, such as ram-pressure stripping, might decrease the observability period of the dark galaxies dramatically. In addition, observations suggest that low-luminosity objects tend to deviate from the BTFR in the opposite direction (McGaugh \& Wolf 2010; Danieli et al. 2020; Müller et al. 2020; Mancera Piña et al. 2020). It is also worth noting that in this scenario, we would expect similar objects to also exist in other clusters, but the current lack of such detections might be an observational bias.

Ram-pressure stripped gas. Gas can also become detached from galaxies in clusters by ram-pressure stripping. In such a scenario, we would expect the HI signal to form a long band with the source galaxy at one end (see, e.g., Jáchym et al. 2014; Kenney et al. 2014). Figure 3 suggests that AGESVC1 282, the dark cloud AGESVC1 247, and the galaxy VCC 740 indeed lie along a line. We note two difficulties with this explanation. First, the band is not contiguous as in the confirmed cases of rampressure stripping. Second, in contradiction with the observed situation, we would expect a monotonic gradient of radial velocity along the gas stream.

Magnetically bound gas cloud. Villaescusa-Navarro et al. (2016) and Nelson et al. (2020) describe populations of optically 
dark neutral gas clouds in cluster simulations, which formed from the cooling and condensation of the hot ICM. In the latter case, the pressure from the magnetic fields within these clouds dominates over their thermal pressure and they can survive on gigayear timescales. Unfortunately we cannot compare their kinematics to the observed Virgo clouds since the simulations do not produce artificial spectra. However, while their size (in excess of $1 \mathrm{kpc}$ ) is consistent with the Virgo clouds, the simulated clouds are at least two orders of magnitude less massive though the parent halo studied is also less massive than the Virgo cluster.

Although there are only a few known observational characteristics of AGESVC1 282, we do not have a theoretical explanation of the nature of the object that would fit them all. Further theoretical and observational investigations of the Virgo dark clouds are obviously needed.

This work used the following scientific software: Astrometry.net (Lang et al. 2010), and SWARP (Bertin 2010). It also employed the following python 3 packages: Astropy (Astropy Collaboration 2013), ccdproc (Craig et al. 2015), and sep (Barbary 2016), which is a python implementation of Source Extractor (Bertin \& Arnouts 1996).

Acknowledgements. We thank the referee for the constructive report, which helped to clarify and improve the manuscript. O.M. is grateful to the Swiss National Science Foundation for financial support. A.V. acknowledges the financial support of the Ministry of Education, Science and Technological Development of the Republic of Serbia (MESTDRS) through the contract No 451-03-68/2020-14/200002 and the financial support by the European Commission through project BELISSIMA (BELgrade Initiative for Space Science, Instrumentation and Modelling in Astrophysics, call FP7-REGPOT-2010-5, contract No. 256772), which was used to procure the Milanković 1.4 meter telescope with the support from the MESTDRS. R.T. acknowledges support from the Czech Ministry of Education, Youth and Sports from the large Infrastructures for Research, Experimental Development and Innovations project LM 2015067, the Czech Science Foundation grant CSF 19-18647S, and the institutional project RVO 67985815. We thank the technical operators at the Astronomical Station Vidojevica (ASV), Miodrag Sekulić and Petar Kostić, for their excellent work.

\section{References}

Alam, S., Albareti, F. D., Allende Prieto, C., et al. 2015, ApJS, 219, 12 Arrigoni Battaia, F., Gavazzi, G., Fumagalli, M., et al. 2012, A\&A, 543, A112 Astropy Collaboration (Robitaille, T. P., et al.) 2013, A\&A, 558, A33 Auld, R., Minchin, R. F., Davies, J. I., et al. 2006, MNRAS, 371, 1617 Bacchini, C., Fraternali, F., Iorio, G., \& Pezzulli, G. 2019, A\&A, 622, A64 Barbary, K. 2016, J. Open Sour. Softw., 1, 58

Bertin, E. 2010, Astrophysics Source Code Library [record ascl:1010.068] Bertin, E., \& Arnouts, S. 1996, A\&AS, 117, 393

Bothun, G. D., Impey, C. D., Malin, D. F., \& Mould, J. R. 1987, AJ, 94, 23

Collins, M. L. M., Tollerud, E. J., Rich, R. M., et al. 2020, MNRAS, 491, 3496

Craig, M. W., Crawford, S. M., Deil, C., et al. 2015, Astrophysics Source Code Library [record ascl:1510.007]
Dabringhausen, J., \& Fellhauer, M. 2016, MNRAS, 460, 4492

Danieli, S., van Dokkum, P., Abraham, R., et al. 2020, ApJ, 895, L4

Davies, J. I., Disney, M. J., Minchin, R. F., Auld, R., \& Smith, R. 2006, MNRAS, 368, 1479

Duc, P.-A., \& Bournaud, F. 2008, ApJ, 673, 787

Gavazzi, G., Boselli, A., Scodeggio, M., Pierini, D., \& Belsole, E. 1999, MNRAS, 304, 595

Greco, J. P., Greene, J. E., Strauss, M. A., et al. 2018, ApJ, 857, 104

Green, D. A. 2011, Bull. Astron. Soc. India, 39, 289

Habas, R., Marleau, F. R., Duc, P.-A., et al. 2020, MNRAS, 491, 1901

Herrmann, K. A., Hunter, D. A., Zhang, H.-X., \& Elmegreen, B. G. 2016, AJ, 152,177

Hess, K. M., Cluver, M. E., Yahya, S., et al. 2017, MNRAS, 464, 957

Impey, C., Bothun, G., Malin, D., \& Staveley-Smith, L. 1990, ApJ, 351, L33

Jáchym, P., Combes, F., Cortese, L., Sun, M., \& Kenney, J. D. P. 2014, ApJ, 792, 11

Javanmardi, B., Martinez-Delgado, D., Kroupa, P., et al. 2016, A\&A, 588, A89

Jester, S., Schneider, D. P., Richards, G. T., et al. 2005, AJ, 130, 873

Kenney, J. D. P., Geha, M., Jáchym, P., et al. 2014, ApJ, 780, 119

Kent, B. R., Spekkens, K., Giovanelli, R., et al. 2009, ApJ, 691, 1595

Koda, J., Yagi, M., Yamanoi, H., \& Komiyama, Y. 2015, ApJ, 807, L2

Koopmann, R. A., Giovanelli, R., Haynes, M. P., et al. 2008, ApJ, 682, L85

Lang, D., Hogg, D. W., Mierle, K., Blanton, M., \& Roweis, S. 2010, AJ, 139, 1782

Leisman, L., Haynes, M. P., Giovanelli, R., et al. 2016, MNRAS, 463, 1692

Mancera Piña, P. E., Fraternali, F., Oman, K. A., et al. 2020, MNRAS, 495, 3636

McGaugh, S. S. 2005, ApJ, 632, 859

McGaugh, S. S., \& Wolf, J. 2010, ApJ, 722, 248

Mihos, J. C. 2019, ArXiv e-prints [arXiv:1909. 09456]

Mihos, J. C., Durrell, P. R., Ferrarese, L., et al. 2015, ApJ, 809, L21

Mihos, J. C., Carr, C. T., Watkins, A. E., Oosterloo, T., \& Harding, P. 2018, ApJ, 863, L7

Minchin, R., Davies, J., Disney, M., et al. 2007, ApJ, 670, 1056

Müller, O., Jerjen, H., \& Binggeli, B. 2017, A\&A, 597, A7

Müller, O., Vudragović, A., \& Bílek, M. 2019, A\&A, 632, L13

Müller, O., Marleau, F. R., Duc, P.-A., et al. 2020, A\&A, 640, A106

Nelson, D., Sharma, P., Pillepich, A., et al. 2020, MNRAS, 498, 2391

Prole, D. J., van der Burg, R. F. J., Hilker, M., \& Davies, J. I. 2019, MNRAS, 488, 2143

Román, J., \& Trujillo, I. 2017, MNRAS, 468, 703

Sandage, A., \& Binggeli, B. 1984, AJ, 89, 919

Sawala, T., Frenk, C. S., Fattahi, A., et al. 2016, MNRAS, 457, 1931

Serra, P., Koribalski, B., Kilborn, V., et al. 2015, MNRAS, 452, 2680

Simon, J. D., \& Geha, M. 2007, ApJ, 670, 313

Simpson, C. M., Grand, R. J. J., Gómez, F. A., et al. 2018, MNRAS, 478, 548

Slater, C. T., Harding, P., \& Mihos, J. C. 2009, PASP, 121, 1267

Taylor, R., Davies, J. I., Auld, R., \& Minchin, R. F. 2012, MNRAS, 423, 787

Taylor, R., Davies, J. I., Auld, R., Minchin, R. F., \& Smith, R. 2013, MNRAS, 428, 459

Taylor, R., Davies, J. I., Jáchym, P., et al. 2016, MNRAS, 461, 3001

Taylor, R., Davies, J. I., Jáchym, P., et al. 2017, MNRAS, 467, 3648

Taylor, R., Wünsch, R., \& Palouš, J. 2018, MNRAS, 479, 377

Taylor, R., Köppen, J., Jáchym, P., et al. 2020, AJ, 159, 218

Trujillo, I., \& Fliri, J. 2016, ApJ, 823, 123

van Dokkum, P. G., Abraham, R., Merritt, A., et al. 2015, ApJ, 798, L45

Venhola, A., Peletier, R., Laurikainen, E., et al. 2017, A\&A, 608, A142

Villaescusa-Navarro, F., Planelles, S., Borgani, S., et al. 2016, MNRAS, 456, 3553 